\title{
Two Meningiomas With Different Histological Grades in the Same Patient -Case Report-
}

\author{
Hakan EMmEZ, Aydemir KALE, Mehmet TÖNGE, \\ Aslı ÇAKIR*, and Necdet ÇEVIKER \\ Departments of Neurosurgery and *Pathology, \\ Gazi University Faculty of Medicine, Ankara, Turkey
}

\begin{abstract}
A 56-year-old female presented with two intracranial meningiomas with different grades manifesting as speech disorder for one week. Neurological and radiological evaluations showed two distinct tumors suggestive of multicentric meningiomas. Surgical resection of both masses was performed in the same procedure. Histological examination found both meningothelial and atypical meningiomas. Concurrency of meningiomas with different grades in same patient is extremely rare. The present case emphasizes the need for follow up of all patients with meningioma even if the neuroimaging features indicate benign character.
\end{abstract}

Key words: atypical meningioma, different histological types, meningothelial meningioma

\section{Introduction}

Meningiomas account for $13 \%$ to $26 \%$ of primary intracranial tumors. Most meningiomas (90\%) arise from neoplastic arachnoidal cells, and have benign character. According to the World Health Organization (WHO) 2000 classification, meningiomas have three main histological subtypes, grades 1,2 , and 3 . Approximately $8 \%$ of meningiomas display multicentric pattern. Concurrent meningiomas in different grades is extremely rare. We describe a case of concurrent WHO grade 1 and 2 meningiomas in the same patient. ${ }^{1,3,5,7)}$

\section{Case Report}

A 56-year-old female who was experiencing trouble while speaking for approximately one week was admitted to our

Received November 10, 2009;

Accepted January 22, 2010 clinic. The patient had also suffered from poor sense of balance in the last 3 years. She had no significant history other than myoma uteri surgery and goiter. Hematological parameters did not indicate any abnormality and neurological evaluation only found mild agitation. $\mathrm{T}_{1}$-weighted magnetic resonance imaging showed an $8 \times 8$-cm mass lesion in the frontal lobe consistent with giant meningioma with a homogeneously enhanced bifrontal dural tail extension (Fig. 1). The meningioma was parafalxian reaching to the skull base. Additionally, another $2.5 \times 3$-cm mass lesion was detected in the right fronto-temporal convexity with a dural tail which was more heterogeneous (Fig. 2).

Total excision of the two lesions including the invaded dura mater was performed in a single session using bicoronal craniotomy. Gross examination found the tumor in the frontal lobe measured $7.5 \times 7 \times 6 \mathrm{~cm}$ and the tumor in the temporal lobe measured $2.5 \times 2.5 \times 2 \mathrm{~cm}$. Both tumors were rubbery and the cut surfaces were grayish white. Histological examination of the resected tumor 

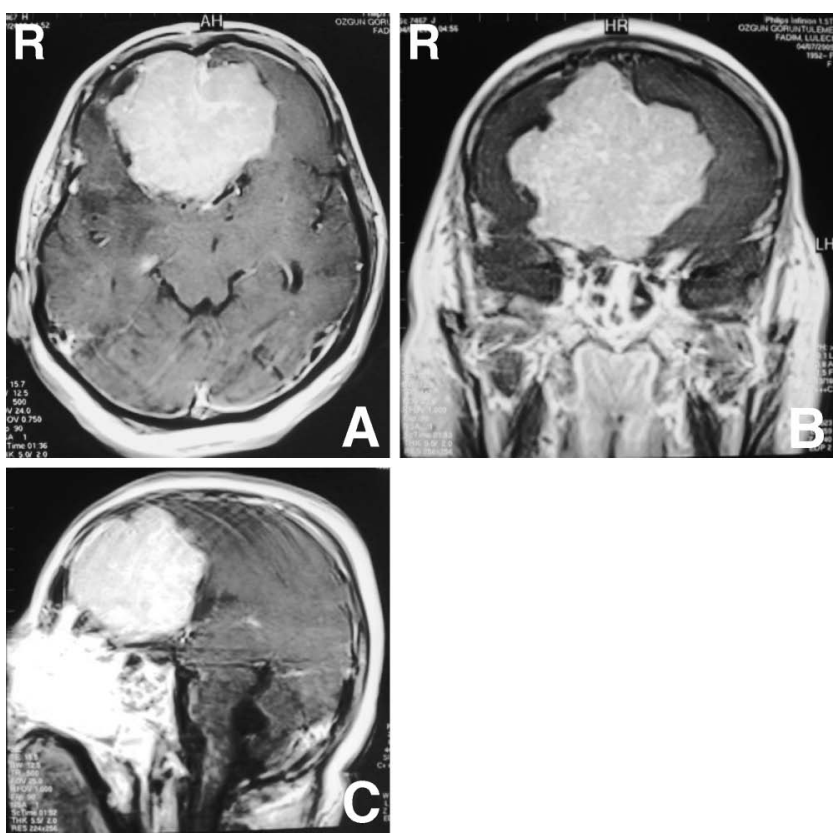

Fig. 1 Preoperative axial (A), coronal (B), and sagittal (C) $T_{1^{-}}$ weighted magnetic resonance images with contrast medium showing a meningioma in the frontal-parafalxian region.
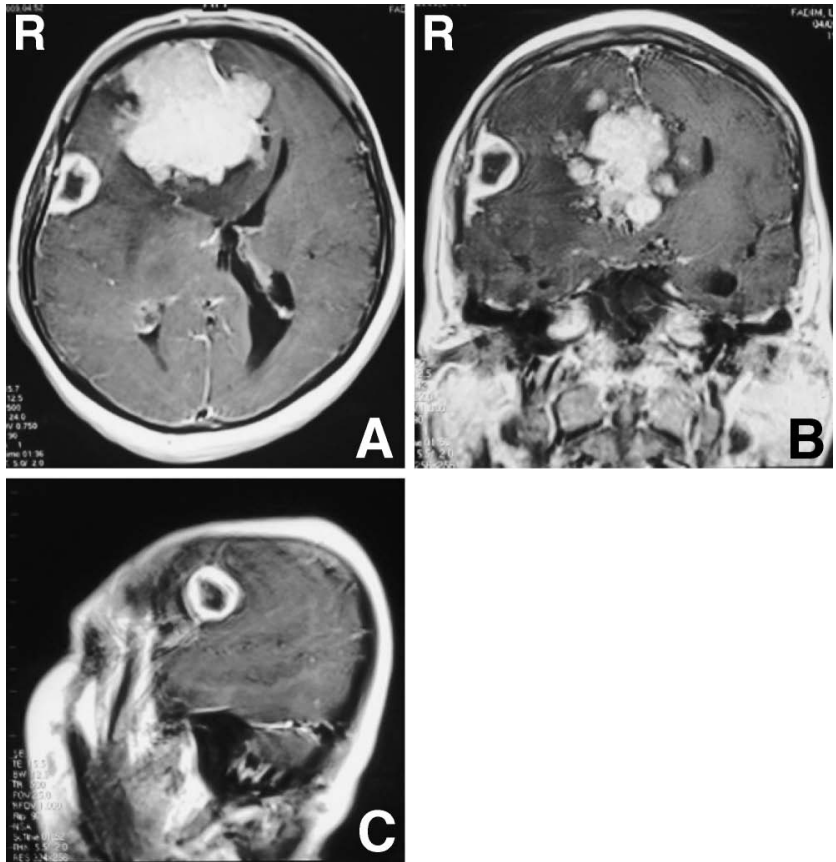

Fig. 2 Preoperative axial (A), coronal (B), and sagittal (C) $T_{1^{-}}$ weighted magnetic resonance images with contrast medium showing a meningioma in the right fronto-temporal region.

from the frontal lobe revealed lobules of cells forming whorls and a few onion bulb structures (Fig. 3). No increase in cellularity and nucleus: cytoplasm ratio or patternless pattern was observed. One mitosis was counted

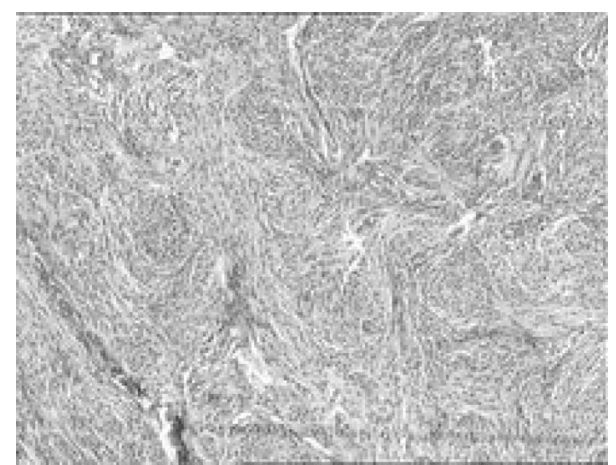

Fig. 3 Photomicrograph of the frontal parafalxian tumor showing tumor cell lobules surrounded by collagenous septa, whorls, and some concentric onion bulb structures. Hematoxylin and eosin stain, $\times 100$.

per 10 high power fields. The histological diagnosis was meningothelial meningoma, WHO grade 1 . Histological examination of the tumor from the temporal lobe showed sheet-like pattern with psammomatous bodies (Fig. 4A). High nucleus:cytoplasmic ratio and prominent nucleoli were seen in the tumor cells. A total of 6 mitoses were identified per 10 high power fields (Fig. 4B, C). The histological diagnosis was atypical meningioma, WHO grade 2 . The patient was discharged without additional neurological deficit and close follow up was recommended (Fig. 5).

\section{Discussion}

Meningiomas are usually benign but can demonstrate multi-centric patterns in $1-10 \%$ of cases in different series. ${ }^{5,7)}$ However, the multi-centricity ratio is higher than previously believed, especially since computed tomography was introduced. The ratio increases to $49 \%$ in surgical cases where tumors are removed at the macroscopic level. ${ }^{1)}$ The multi-centricity ratio was $8.2 \%$ in 231 incidental meningioma cases in a very extensive cadaver study. ${ }^{4}$

Almost all cases of multiple meningioma demonstrate common features. There are several hypotheses regarding their development, but the most widely accepted hypothesis asserts that tumoral cell clones form the second mass lesion by spreading across the meninges. In this hypothesis, movement through the cerebrospinal fluid and even hematogenous expansion are proposed. Rarely, multiple meningiomas display different sporadic patterns in the same patient. Hypotheses supporting this pattern assume the concurrent development of independent tumors. ${ }^{2,5}$ One case included atypical and psammomatous meningiomas in different locations. ${ }^{3)}$ Another study described concurrent fibrous and atypical meningiomas in different intracranial locations. ${ }^{6}$ The present case included concurrent meningothelial and atypical meningiomas.

Multimodal treatment approaches are possible for meningiomas. Treatment is usually carried out with respect to the histological classification. WHO grade 1 meningiomas generally require total excision of the lesion 


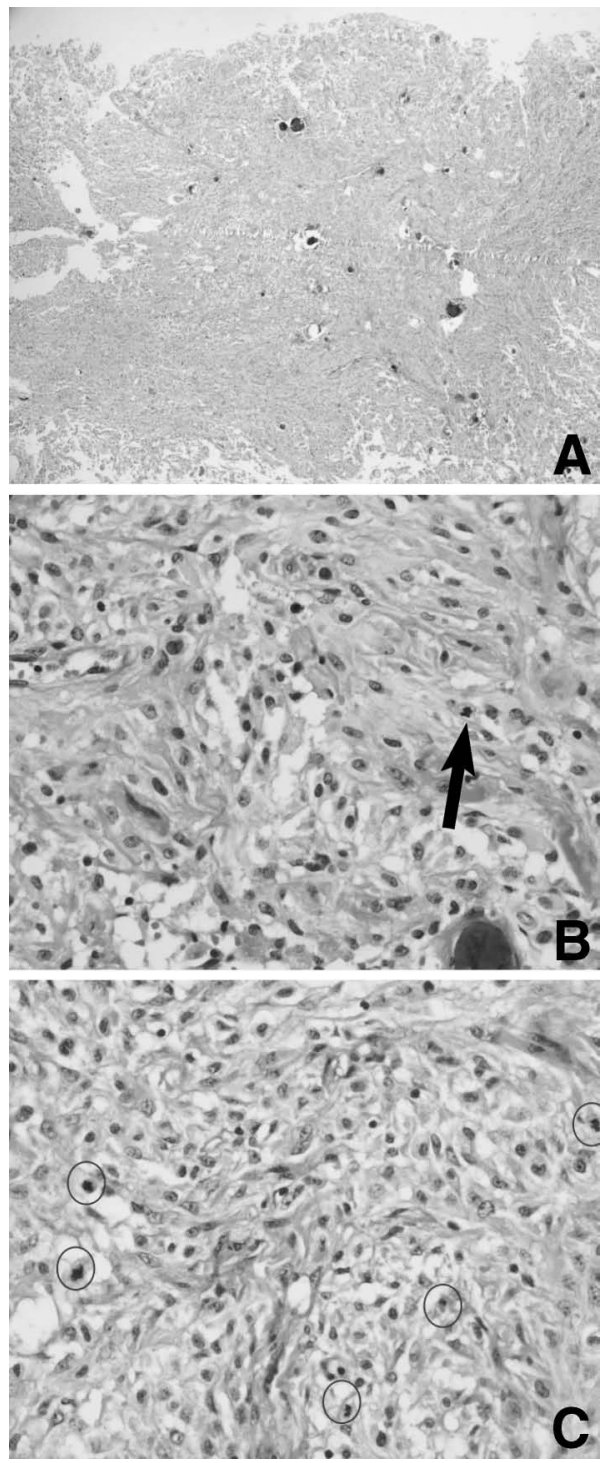

Fig. 4 Photomicrographs of the fronto-temporal tumor tissue showing meningothelial cells with sheet-like pattern and psammomatous bodies (A: hematoxylin and eosin stain, $\times 140$ ), prominent nucleoli and a mitotic figure (arrow) (B: hematoxylin and eosin stain, $\times 400$ ), and 5 mitoses in one high power field (C: hematoxylin and eosin stain, $\times 400$ ).

with dural extension (Simpson grade 1). WHO grade 2 and especially WHO grade 3 meningiomas have malignant character, so clinical and radiological follow up is required. Additional treatment regimens such as secondary surgery, radiotherapy, and radiosurgery may be necessary. The presented case was closely followed up due to the grade 2 meningioma. Any small secondary lesion distant from primary craniotomy region would only be followed up and would only be treated in the follow-up period. The present case is an important example which emphasizes

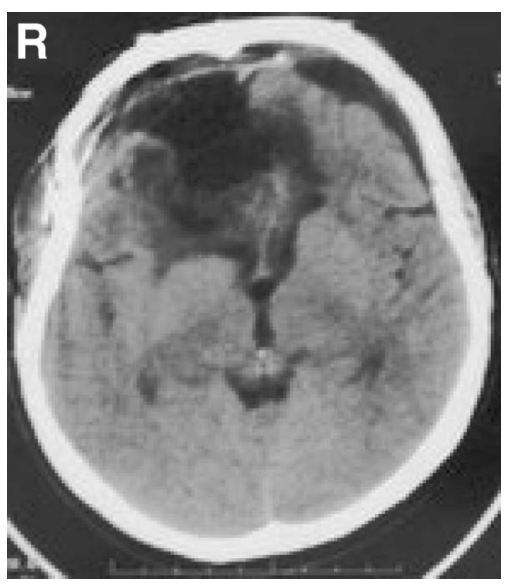

Fig. 5 Precontrast computed tomography scan taken on the 3rd day after operation.

the need for follow up of all patients with meningioma even if the neuroimaging features indicate benign character. Multiple meningiomas are not uncommon in neurofibromatosis type 2 patients, but are uncommon in sporadic patients, and may very rarely have different histological grades as in our case. Keeping this possibility in mind will be beneficial in the follow up of patients with meningiomas.

\section{References}

1) Borovich B, Doron $Y$, Braun J, Feinsod M, Goldsher D, Gruszkiewicz J, Guilburd JN, Zaaroor M, Levi L, Soustiel JF, Lemberger A: The incidence of multiple meningiomas-do solitary meningiomas exist? Acta Neurochir (Wien) 90: 15-22, 1988

2) de Vries J, Wakhloo AK: Repeated multifocal recurrence of grade I, grade II and grade III meningiomas: regional multicentricity (primary new growth) or metastases? Surg Neurol 41: 299-305, 1994

3) Koh YC, Yoo H, Whang GC, Kwon OK, Park HI: Multiple meningiomas of different patological features: case report. $J$ Clin Neurosci 8 Suppl 1: 40-43, 2001

4) Nakasu S, Hirano A, Shimura T, Llena JF: Incidental meningiomas in autopsy study. Surg Neurol 27: 319-322, 1987

5) Ojo A, Fynn E: Multiple meningiomas: case report. South African Journal of Radiology 10: 21-23, 2006

6) Tomita T, Kurimoto M, Yamatani K, Nagai S, Kuwayama N, Hirashima Y, Endo S: Multiple meningiomas consisting of fibrous meningioma and anaplastic meningioma. J Clin Neurosci 10: 622-624, 2003

7) Whittle IR, Smith C, Navoo P, Collie D: Meningiomas. Lancet 363: 1535-1543, 2004

Address reprint requests to: Aydemir Kale, M.D., Gazi Üniversitesi Tıp Fakültesi, Beyin ve Sinir Cerrahisi AD 1. Kat, 06500 , Ankara, Turkey.

e-mail: aydemirkale@gmail.com 